\title{
Ischemic Cardiomyopathy versus Non-Ischemic Dilated Cardiomyopathy in Patients with Reduced Ejection Fraction- Clinical Characteristics and Prognosis Depending on Heart Failure Etiology (Data from European Society of Cardiology Heart Failure Registries)
}

\author{
Agata Tymińska ${ }^{1}$, Krzysztof Ozierański ${ }^{1, * \mathbb{D}}$, Paweł Balsam ${ }^{1}$, Cezary Maciejewski ${ }^{1}$, Anna Wancerz ${ }^{1}$, \\ Emil Brociek $^{1}{ }^{(\mathbb{D}}$, Michał Marchel ${ }^{1}$ (D) Maria G. Crespo-Leiro ${ }^{2}{ }^{\mathbb{D}}$, Aldo P. Maggioni ${ }^{3}$ (D) Jarosław Drożdz ${ }^{4}$,

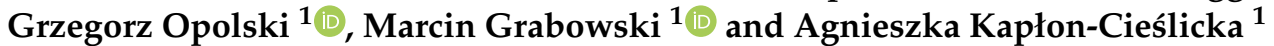

Citation: Tymińska, A.; Ozierański,

K.; Balsam, P.; Maciejewski, C.;

Wancerz, A.; Brociek, E.; Marchel, M.; Crespo-Leiro, M.G.; Maggioni, A.P.; Drożdż, J.; et al. Ischemic Cardiomyopathy versus NonIschemic Dilated Cardiomyopathy in Patients with Reduced Ejection Fraction-Clinical Characteristics and Prognosis Depending on Heart Failure Etiology (Data from European Society of Cardiology Heart Failure Registries). Biology 2022, 11, 341.

https://doi.org/10.3390/

biology11020341

Academic Editors: Zhongjian Cheng and Lemin Zheng

Received: 1 January 2022

Accepted: 14 February 2022

Published: 21 February 2022

Publisher's Note: MDPI stays neutral with regard to jurisdictional claims in published maps and institutional affiliations.

Copyright: (C) 2022 by the authors. Licensee MDPI, Basel, Switzerland. This article is an open access article distributed under the terms and conditions of the Creative Commons Attribution (CC BY) license (https:// creativecommons.org/licenses/by/ $4.0 /)$
1 First Department of Cardiology, Medical University of Warsaw, 02-097 Warsaw, Poland; agata.tyminska@wum.edu.pl (A.T.); pawel.balsam@wum.edu.pl (P.B.); cmaciejewski6@gmail.com (C.M.); anna.wancerz22@gmail.com (A.W.); emil.brociek@gmail.com (E.B.); michal.marchel@wum.edu.pl (M.M.); grzegorz.opolski@wum.edu.pl (G.O.); marcin.grabowski@wum.edu.pl (M.G.); agnieszka.kaplon@gmail.com (A.K.-C.)

2 Instituto de Investigaci on Biomedica de A Coruña (INIBIC), Complexo Hospitalario Universitario A Coruña (CHUAC)-CIBERCV, 15006 La Coruña, Spain; marisacrespo@gmail.com

3 Centro Studi ANMCO (Associazione Nazionale Medici Cardiologi Ospedalieri), 50121 Florence, Italy; maggioni@anmco.it

4 2nd Department of Cardiology, Central University Hospital, Medical University of Lodz, 92-213 Łódź, Poland; jaroslaw.drozdz@umed.pl

* Correspondence: krzysztof.ozieranski@wum.edu.pl; Tel.: +48-22-5992958; Fax: +48-22-5991957

Simple Summary: Given the high morbidity and mortality linked with heart failure and the need for disease-specific treatment, there is international agreement that there is a significant need for well-planned, large-scale databases showing the true course of heart failure. We present a study based on data from the European Society of Cardiology Heart Failure registries designed to evaluate the prevalence, clinical characteristics, management and outcomes of patients with two main etiologies of heart failure: reduced left ventricular ejection fraction-ischemic cardiomyopathy (ICM) and nonischemic dilated cardiomyopathy (NIDCM). Our findings show that the patients with ICM were older and had more comorbidities. In contrast, the patients with NIDCM had worse systolic heart function. Apart from the more frequent use of aldosterone antagonists in the NIDCM group, there were no other differences as regards the use of heart failure guideline-recommended medications, implantable cardioverter defibrillators or cardiac resynchronization therapy. One-year prognosis was worse in the ICM patients than in the NIDCM patients. Moreover, ICM etiology itself was associated with a worse one-year outcome.

Abstract: Personalized management involving heart failure (HF) etiology is crucial for better prognoses for HF patients. This study aimed to compare patients with ischemic cardiomyopathy (ICM) and patients with non-ischemic dilated cardiomyopathy (NIDCM) in terms of baseline characteristics and prognosis. We assessed 895 patients with HF with reduced left ventricular ejection fraction participating in the Polish part of the European Society of Cardiology (ESC)-HF registries. ICM was present in 583 patients $(65 \%)$, NIDCM in 312 patients $(35 \%)$. The ICM patients were older $(p<0.001)$ and had more comorbidities. The NIDCM patients more frequently had atrial fibrillation $(p=0.04)$ and lower LVEF ( $p=0.01)$; therefore, they were treated more often with anticoagulants $(p=0.01)$ and digitalis $(p<0.001)$. The NIDCM patients were prescribed aldosterone antagonists more often $(p=0.01)$. There were no other differences as regards the use of HF guideline-recommended medications, implantable cardioverter defibrillators or cardiac resynchronization therapy. The ICM patients were more likely to be treated with statins $(p<0.001)$ and antiplatelet agents $(p<0.001)$ All-cause death, as well as all-cause death and readmissions for HF at 12 months, occurred more often 
in the ICM group compared with the NIDCM group (15.9\% vs. $10 \%, p=0.016$; and $40.9 \%$ vs. $28.6 \%$, $p=0.00089$, respectively). ICM etiology was an independent predictor of the composite endpoint in the total cohort $(p=0.003)$. The ICM patients were older and had more comorbidities, whereas the NIDCM patients had lower LVEF. One-year prognosis was worse in the ICM patients than in the NIDCM patients. ICM etiology was independently associated with a worse one-year outcome.

Keywords: personalized management; coronary artery disease; atherosclerosis; heart failure; mortality

\section{Introduction}

Heart failure (HF) incidence and morbidity are on the increase, with a HF prevalence of approximately $1-2 \%$ in adults in developed countries, rising to $\geq 10 \%$ in patients aged 70 years or over $[1,2]$. It is estimated that of those approximately $50 \%$ suffer from HF with reduced ejection fraction (HFrEF). Although there are well-established therapies for HFrEF that help to improve symptoms, quality of life and outcomes, the overall prognosis in HF patients remains poor as the 5-year mortality rate after diagnosis is approximately $50 \%[1,3,4]$. It is estimated that around $40 \%$ of patients hospitalized for HF will die or will be rehospitalized within a year, with the highest frequency of hospital readmissions in the early post-discharge period [4,5]. Therefore, the current focus of HF therapy is shifting towards a better assessment of the underlying HF etiology and personalized patient management. According to the guidelines of the European Society of Cardiology (ESC), establishing HF etiology should constitute the initial step, crucial for planning an appropriate therapy [1]. However, knowledge about clinical differences and their impact on the prognosis in ischemic cardiomyopathy (ICM) versus non-ischemic dilated cardiomyopathy (NIDCM) patients remains unsatisfactory.

The underlying pathophysiology of both forms of cardiomyopathy is different (i.e., atherosclerosis, inflammation or genetic) and this subsequently may influence the clinical characteristics, the course of the disease and the prognosis, and therefore the management of these patients. Previous studies have reported conflicting results in terms of mortality risk in patients with ICM and NIDCM [6-10]. Patients with ICM are at a greater risk of sudden cardiac death (SCD) than patients with NIDCM; thus, the benefits resulting from implantable cardioverter defibrillators (ICDs) are greater in the first group [1,11]. What is more, a recently published nationwide study has shown one-year mortality to be significantly higher in ICM than in NIDCM patients after ICD implantation for primary prevention of SCD [12]. However, current therapeutic strategies and risk assessments are imperfect, as they are mostly based on the left ventricular ejection fraction (LVEF) and the New York Heart Association Classification (NYHA) and do not account for the underlying HF etiology. Still more data, particularly from real-world patient studies, are necessary to improve etiology-based HF management.

In this study we evaluate the prevalence of ICM and NIDCM etiology as well as the associated clinical characteristics and the prognosis in HFrEF patients.

\section{Patients and Methods}

\subsection{Study Design}

The study involved data from the ESC-HF Pilot and the ESC-HF Long-Term registries of the European Society of Cardiology (ESC). These registries are based on multicenter, prospective, observational surveys lasting from October 2009 to May 2010 in 136 European cardiology centers (including 29 centers in Poland) and from April 2011 to January 2015 in 211 European cardiology centers (including 35 centers in Poland), respectively. Eligible patients were enrolled into the study if they were at least 18 years of age and met the diagnostic criteria for HF, including both outpatients and inpatients with chronic, worsening or new-onset HF. There were no other specific exclusion criteria. The current study included Polish patients of the ESC-HF Pilot Survey and of the ESC-HF Long-Term Registry. The 
study protocol was approved by the local ethics committees. All participating patients were provided with detailed information and signed informed consent for the study. Records collected in both registries included clinical characteristics, test results, HF management and one-year follow-up. A detailed study design was published previously $[13,14]$.

The current analysis included both ambulatory and hospitalized HFrEF patients (LVEF $<40 \%$ ). The study participants were divided into two groups based on HF etiology -ICM or NIDCM. The ICM group included patients with coronary artery disease as the primary cause of HF, whereas the NIDCM group included patients primarily considered as presenting with dilated cardiomyopathy based on the enrolling investigators' opinion according to actual knowledge. Patients with a strong etiological factor, i.e., significant valve disease, tachycardia-induced cardiomyopathy and with LVEF $\geq 40 \%$ were excluded from the analysis.

\subsection{Study Endpoints}

The primary endpoint was all-cause death at one year. The secondary endpoint was a composite of all-cause death and hospitalization for HF worsening at one year. NYHA class after one year was also analyzed.

Additionally, we sought to determine the clinical characteristics and predictors of one-year outcomes in the studied groups.

\subsection{Statistical Analysis}

The results were presented as medians and quartiles for continuous variables and as frequencies and percentages for categorical and ordinal variables. The frequencies of the categorical and ordinal variables in the groups were compared by Fisher's exact test and the continuous variables by Mann-Whitney U test, respectively. Kaplan-Meier survival curves were plotted for both study endpoints. Cox proportional hazards regression models were used to identify predictors of the primary and secondary endpoints. Analyses for both study-points regarding HF etiology were performed, adjusting for age, LVEF and NYHA class at baseline, in order to account for important differences in the studied groups. The selection of the potential predictors of outcomes for ICM and NIDCM etiology of HF was guided by background knowledge on the topic. For the selected variables a series of univariable analyses was performed. Variables with the $p$-values below $<0.10$ threshold in univariable analyses were included in the multivariable analyses. A $p$-value below 0.05 was considered significant for all tests. All tests were two-tailed. Statistical analyses were performed using R software, version 3.6.2 (R Core Team 2020, R Foundation for Statistical Computing, Vienna, Austria).

\section{Results}

\subsection{Study Group Selection}

Figure 1 shows the flow chart of patient selection for the present study. The ESC-HF Pilot and the ESC-HF Long-Term registries comprised 5118 and 12,440 patients across Europe, respectively. The total Polish cohort of the registry consisted of 2019 patients, including 1415 inpatients and 604 outpatients. Out of them, 895 patients (616 inpatients and 279 outpatients) with HFrEF were included in this study. Among those, 583 (65.1\%) and $312(34.9 \%)$ patients had ICM and NIDCM, respectively.

\subsection{Clinical Characteristics}

The ICM patients were older (median: 67 vs. 58 years) and with a higher LVEF ( $28 \%$ vs. $25 \%)$ compared with the NIDCM patients. The ICM patients also had more comorbidities (i.e., hypertension, peripheral artery disease, diabetes, chronic kidney disease, chronic obstructive pulmonary disease, previous stroke or transient ischemic attack) than the NIDCM patients. More patients in the ICM group declared current use of tobacco, whereas the NIDCM group more frequently reported alcohol usage. More patients in the NIDCM group had atrial fibrillation; thus, they were more frequently treated with 
anticoagulants and digitalis. Moreover, the patients with NIDCM received aldosterone antagonists more frequently. There were no other differences as regards the use of HF guideline-recommended medications or ICD/cardiac resynchronization therapy (CRT). Due to the ischemic etiology, the ICM patients were more likely to be treated with statins and antiplatelet agents. Detailed baseline characteristics of the study groups are presented in Tables 1 and 2.

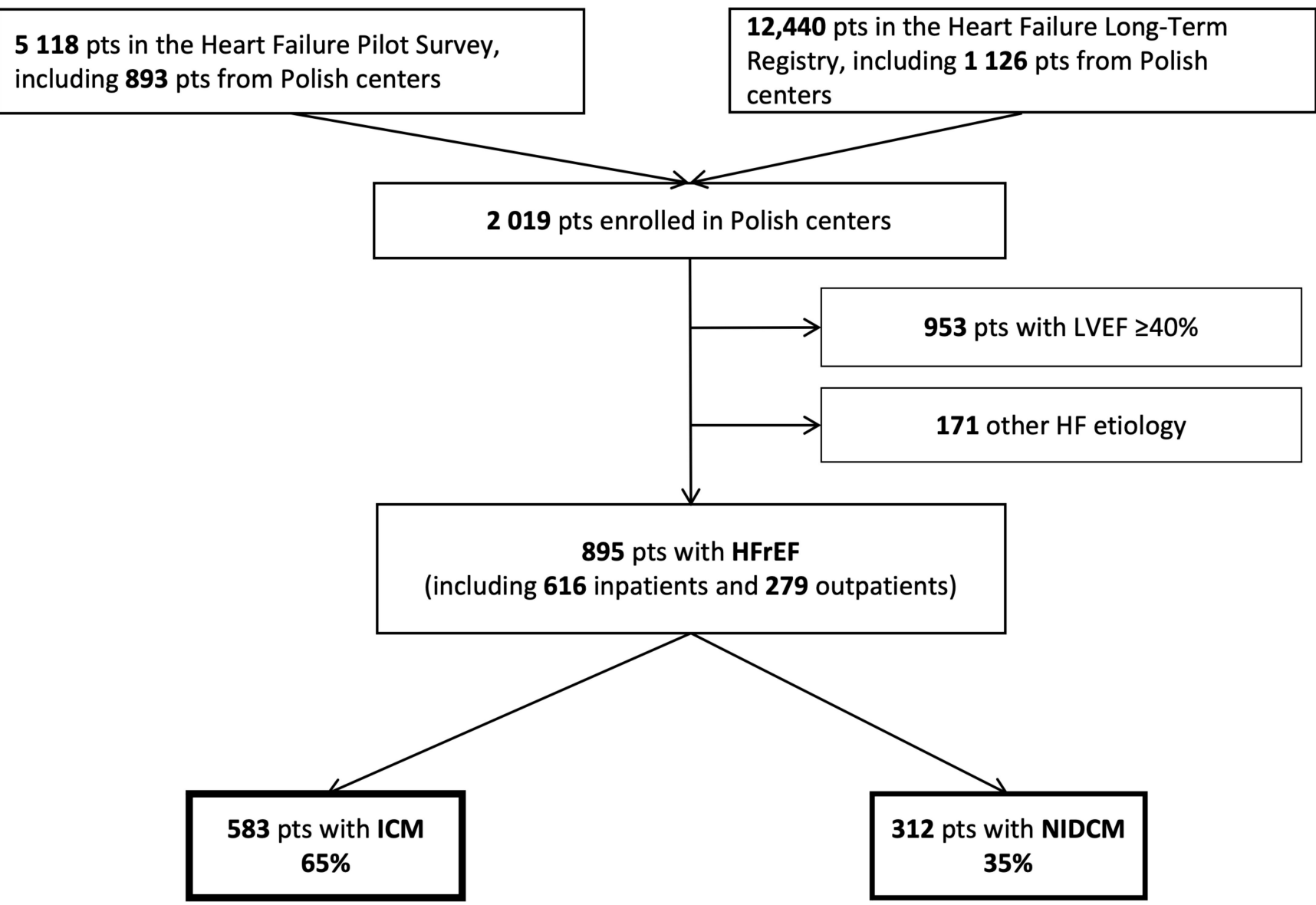

Figure 1. Flow chart of patient enrollment in the current analysis.

\subsection{One-Year Outcomes and Clinical Predictors}

The primary and secondary endpoints occurred more frequently in the ICM group compared with the NIDCM group (15.9\% vs. $10 \%, p=0.016$; and $40.9 \%$ vs. $28.6 \%, p=0.00089$, respectively) (Table 1). Kaplan-Meier curves of both study endpoints are plotted in Figures 2 and 3. In addition, Kaplan-Meier curves of both study endpoints after censoring for in-hospital events are included in the Supplementary Materials (Figures S1 and S2). There was no difference between the groups as regards the NYHA class after one-year $(p=0.15)$. 
Table 1. Baseline characteristics and clinical outcomes in total cohort of HF patients and ICM or NIDCM.

\begin{tabular}{|c|c|c|c|}
\hline & \multicolumn{2}{|c|}{ HFrEF Patients $(n=895)$} & \multirow[b]{2}{*}{$p$-Value } \\
\hline & $\begin{array}{c}\text { ICM } \\
(n=583)\end{array}$ & $\begin{array}{l}\text { NIDCM } \\
(n=312)\end{array}$ & \\
\hline \multicolumn{4}{|c|}{ Baseline characteristics } \\
\hline Age, years & $66.5(58.7-75.2)$ & $58.2(49.3-65.2)$ & $<0.001$ \\
\hline Male & $468(80.3 \%)$ & $255(81.7 \%)$ & 0.66 \\
\hline Previous hospitalization & $350(61.2 \%) ; n=572$ & $179(57.9 \%) ; n=309$ & 0.35 \\
\hline $\mathrm{BMI}, \mathrm{kg} / \mathrm{m}^{2}$ & $27.30(24.70-30.10) ; n=554$ & $27.80(25.00-31.80) ; n=310$ & 0.01 \\
\hline Current LVEF, \% & $28(20-33)$ & $25(20-30)$ & 0.01 \\
\hline Previous HF hospitalization & $433(74.3 \%)$ & $183(58.7 \%)$ & $<0.001$ \\
\hline Prior PCI or CABG & $424(72.7 \%)$ & $0(0.0 \%)$ & $<0.001$ \\
\hline Moderate or severe mitral regurgitation & $302(57.7 \%) ; n=523$ & $149(54.2 \%) ; n=275$ & 0.37 \\
\hline Moderate or severe aortic stenosis & $16(3.1 \%) ; n=523$ & $7(2.6 \%) ; n=272$ & 0.83 \\
\hline Moderate or severe aortic regurgitation & $41(7.8 \%) ; n=524$ & $13(4.8 \%) ; n=272$ & 0.13 \\
\hline Moderate or severe tricuspid regurgitation & $182(34.8 \%) ; n=523$ & $109(40.1 \%) ; n=272$ & 0.16 \\
\hline LVEDD, mm & $63.0(58.0-70.0) ; n=506$ & $67.0(60.5-75.0) ; n=267$ & $<0.001$ \\
\hline LBBB & $89(17.0 \%) ; n=524$ & $59(21.8 \%) ; n=271$ & 0.10 \\
\hline QRS, ms & $114.5(100.0-139.2) ; n=496$ & $118.0(100.0-141.0) ; n=255$ & 0.48 \\
\hline Hypertension & $400(68.7 \%) ; n=582$ & $112(36.1 \%) ; n=310$ & $<0.001$ \\
\hline History of atrial fibrillation & $201(34.5 \%)$ & $130(41.8 \%) ; n=311$ & 0.04 \\
\hline Peripheral artery disease & $97(16.7 \%) ; n=582$ & $13(4.2 \%)$ & $<0.001$ \\
\hline Diabetes & $233(40.0 \%)$ & $82(26.3 \%)$ & $<0.001$ \\
\hline Chronic kidney disease & $140(24.0 \%)$ & $42(13.5 \%)$ & $<0.001$ \\
\hline COPD & $121(20.8 \%)$ & $40(12.8 \%)$ & 0.01 \\
\hline Prior stroke or TIA & $78(13.4 \%)$ & $24(7.7 \%)$ & 0.01 \\
\hline Current or former smoking & $406(70.7 \%) ; n=574$ & $198(63.7 \%) ; n=311$ & 0.03 \\
\hline Alcohol usage & $331(60.5 \%) ; n=547$ & $210(70.7 \%) ; n=297$ & 0.01 \\
\hline Pacemaker & $30(5.1 \%)$ & $9(2.9 \%)$ & 0.13 \\
\hline ICD & $168(28.8 \%)$ & $102(32.7 \%)$ & 0.25 \\
\hline CRT & $61(10.5 \%)$ & $36(11.5 \%)$ & 0.65 \\
\hline \multicolumn{4}{|c|}{ Clinical status and laboratory findings } \\
\hline Heart rate, b.p.m. & $75.0(67.0-92.0) ; n=581$ & $80.0(70.0-97.8)$ & 0.04 \\
\hline $\mathrm{SBP}, \mathrm{mmHg}$ & $115(105-125) ; n=582$ & $115(105-130)$ & 0.82 \\
\hline $\mathrm{DBP}, \mathrm{mmHg}$ & $70(60-80) ; n=581$ & $70(70-80)$ & $<0.001$ \\
\hline NYHA class & $n=579$ & $n=312$ & 0.01 \\
\hline I & $11(1.9 \%)$ & $14(4.5 \%)$ & - \\
\hline II & $177(30.6 \%)$ & $119(38.1 \%)$ & - \\
\hline III & $254(43.9 \%)$ & $117(37.5 \%)$ & - \\
\hline IV & $137(23.7 \%)$ & $62(19.9 \%)$ & - \\
\hline
\end{tabular}


Table 1. Cont.

\begin{tabular}{|c|c|c|c|}
\hline & \multicolumn{2}{|c|}{ HFrEF Patients $(n=895)$} & \multirow[b]{2}{*}{$p$-Value } \\
\hline & $\begin{array}{c}\text { ICM } \\
(n=583)\end{array}$ & $\begin{array}{l}\text { NIDCM } \\
(n=312)\end{array}$ & \\
\hline Hemoglobin, g/dL & $13.4(12.2-14.7) ; n=535$ & $14.2(13.0-15.2) ; n=259$ & $<0.001$ \\
\hline Serum creatinine, $\mathrm{mg} / \mathrm{dL}$ & $1.1(0.9-1.4) ; n=550$ & $1.1(0.9-1.3) ; n=268$ & 0.01 \\
\hline $\mathrm{eGFR}, \mathrm{mL} / \mathrm{min} / 1.73 \mathrm{~m}^{2}$ & $68.9(48.1-93.8) ; n=550$ & $86.3(60.0-113.7) ; n=268$ & 0.01 \\
\hline Serum sodium, mmol/L & $138.8(136.0-141.0) ; n=547$ & $139.0(136.0-141.0) ; n=263$ & 0.803 \\
\hline NT-proBNP & $3566.0(1575.0-7654.2) ; n=170$ & $2724.0(793.0-5227.0) ; n=97$ & 0.014 \\
\hline \multicolumn{4}{|c|}{ Pharmacotherapy (at discharge) } \\
\hline Diuretics & $508(87.3 \%) n=582$ & $281(90.4 \%)$ & 0.19 \\
\hline Aldosterone antagonist & $429(73.7 \%) ; n=582$ & $254(81.7 \%)$ & 0.01 \\
\hline ACE-I & $450(77.3 \%) ; n=582$ & $254(81.4 \%)$ & 0.17 \\
\hline $\mathrm{ARB}$ & $54(9.3 \%) ; n=582$ & $35(11.3 \%)$ & 0.35 \\
\hline$\beta$-blocker & $540(92.8 \%) ; n=582$ & $294(94.2 \%)$ & 0.48 \\
\hline Statins & $492(84.5 \%) ; n=582$ & $154(49.4 \%)$ & $<0.001$ \\
\hline Anticoagulants & $213(36.7 \%) ; n=581$ & $146(46.8 \%)$ & 0.01 \\
\hline Antiplatelets & $466(80.1 \%) ; n=582$ & $125(40.1 \%)$ & $<0.001$ \\
\hline Digitalis & $135(23.2 \%) ; n=582$ & $112(35.9 \%)$ & $<0.001$ \\
\hline Amiodarone & $74(12.7 \%) ; n=582$ & $50(16.0 \%)$ & 0.18 \\
\hline Antiarrhytmics & $38(6.5 \%) ; n=582$ & $13(4.2 \%)$ & 0.17 \\
\hline $\mathrm{CCB}$ & $50(8.6 \%) ; n=582$ & $9(2.9 \%)$ & 0.001 \\
\hline \multicolumn{4}{|c|}{ One-year outcome } \\
\hline NYHA & $n=447$ & $n=254$ & 0.14 \\
\hline I & $37(8.3 \%)$ & $35(13.8 \%)$ & - \\
\hline II & $251(56.2 \%)$ & $137(53.9 \%)$ & - \\
\hline III & $137(30.6 \%)$ & $72(28.3 \%)$ & - \\
\hline IV & $22(4.9 \%)$ & $10(3.9 \%)$ & - \\
\hline Death & $88(15.9 \%) ; n=555$ & $30(10.0 \%) ; n=301$ & 0.02 \\
\hline Death or rehospitalization & $205(40.9 \%) ; n=501$ & $80(28.6 \%) ; n=280$ & 0.001 \\
\hline
\end{tabular}

If missing data for the respective variable is present, available cases counts are presented in italics. Bolded text indicates $p$ values $<0.05$. ACE-I—angiotensin-converting enzyme inhibitor; ARB—angiotensin receptor blocker; BMIbody mass index; b.p.m. - beats per minute; CABG—coronary artery bypass grafting; CCB — calcium channel blocker; COPD—chronic obstructive pulmonary disease; CRT—cardiac resynchronization therapy; DBP—diastolic blood pressure; $\mathrm{eGFR}$ - estimated glomerular filtration rate; HF-heart failure; ICD-implantable cardioverterdefibrillator; LBBB — left bundle branch block; LVEDD—left ventricular end-diastolic diameter; LVEF-left ventricular ejection fraction; NIDCM-non-ischemic dilated cardiomyopathy; NT-proBNP-N-terminal pro brain natriuretic peptide; NYHA-New York Heart Association; HFrEF-heart failure with reduced ejection fraction PCI—percutaneous coronary intervention; SBP—systolic blood pressure; TIA—-transient ischemic attack.

In the multivariable analysis, the ICM etiology was independently associated with the secondary endpoint $(1.56(1.16-2.11), p=0.003)$ but not with the primary endpoint in the total cohort (Table 3). Independent predictors of the primary and secondary endpoints in both groups are presented in Table 4. 
Table 2. Clinical course of index hospitalization and in-hospital outcomes in HF patients with ICM or NIDCM (only hospitalized participants).

\begin{tabular}{|c|c|c|c|}
\hline & \multicolumn{2}{|c|}{ HFrEF Patients $(n=616)$} & \multirow[b]{2}{*}{$p$-Value } \\
\hline & $\begin{array}{c}\text { ICM } \\
(n=433)\end{array}$ & $\begin{array}{l}\text { NIDCM } \\
(n=183)\end{array}$ & \\
\hline \multicolumn{4}{|c|}{ Clinical status at hospital admission } \\
\hline Cardiogenic shock & $13 / 427(3.1 \%) ; n=417$ & $9 / 175(5.1 \%) ; n=175$ & 0.24 \\
\hline Heart rate, b.p.m. & $80.0(70.0-100.0) ; n=432$ & $86.0(73.5-105.0)$ & 0.01 \\
\hline $\mathrm{SBP}, \mathrm{mmHg}$ & $120.0(110.0-140.0) ; n=432$ & $120.0(109.5-133.5)$ & 0.04 \\
\hline $\mathrm{DBP}, \mathrm{mmHg}$ & $80(70-84) ; n=431$ & $76(70-80)$ & 0.43 \\
\hline NYHA & $n=429$ & $n=183$ & 0.96 \\
\hline I & $3(0.7 \%)$ & $1(0.5 \%)$ & - \\
\hline II & $92(21.4 \%)$ & $39(21.3 \%)$ & - \\
\hline III & $201(46.9 \%)$ & $83(45.4 \%)$ & - \\
\hline IV & $133(31.0 \%)$ & $60(32.8 \%)$ & - \\
\hline Pacemaker & $25(5.8 \%)$ & $7(3.8 \%)$ & 0.43 \\
\hline CRT & $36(8.3 \%)$ & $17(9.3 \%)$ & 0.75 \\
\hline ICD & $112(25.9 \%)$ & $52(28.4 \%)$ & 0.55 \\
\hline \multicolumn{4}{|c|}{ Laboratory findings at admission } \\
\hline Hemoglobin, g/dL & $13.3(12.1-14.7) ; n=425$ & $13.9(12.8-15.1) ; n=181$ & $<0.001$ \\
\hline Serum creatinine, $\mathrm{mg} / \mathrm{dL}$ & $1.1(0.9-1.5) ; n=428$ & $1.1(0.9-1.3) ; n=181$ & 0.02 \\
\hline $\mathrm{eGFR}, \mathrm{mL} / \mathrm{min} / 1.73 \mathrm{~m}^{2}$ & $64.6(45.6-89.2) ; n=428$ & $80.5(58.2-110.7) ; n=181$ & $<0.001$ \\
\hline Serum sodium, $\mathrm{mmol} / \mathrm{L}$ & $138.0(136.0-141.0) ; n=431$ & $138.0(136.0-140.5) ; n=182$ & 0.88 \\
\hline Serum potassium, $\mathrm{mmol} / \mathrm{L}$ & $4.4(4.1-4.8) ; n=430$ & $4.5(4.2-4.8) ; n=182$ & 0.22 \\
\hline \multicolumn{4}{|c|}{ Management during index hospitalization } \\
\hline Inotropic support & $65(15.0 \%)$ & $40(22.0 \%)$ & 0.046 \\
\hline Diuretic i.v. & $300(69.8 \%) ; n=430$ & $120(65.6 \%)$ & 0.34 \\
\hline Nitrates i.v. & $63(14.6 \%) ; n=431$ & $26(14.2 \%)$ & 1.0 \\
\hline \multicolumn{4}{|c|}{ Clinical status and laboratory findings at discharge } \\
\hline Heart rate, b.p.m. & $70(65-78) ; n=420$ & $72(68-80) n=173$ & 0.001 \\
\hline $\mathrm{SBP}, \mathrm{mmHg}$ & $115.0(105.0-120.0) ; n=423$ & $115.0(100.0-120.8) ; n=176$ & 0.81 \\
\hline $\mathrm{DBP}, \mathrm{mmHg}$ & $70(60-80) ; n=422$ & $70(65-80) ; n=176$ & 0.13 \\
\hline NYHA & $n=424$ & $n=176$ & 0.04 \\
\hline I & $27(6.4 \%)$ & $6(3.4 \%)$ & - \\
\hline II & $219(51.7 \%)$ & $111(63.1 \%)$ & - \\
\hline III & $167(39.4 \%)$ & $53(30.1 \%)$ & - \\
\hline IV & $11(2.6 \%)$ & $6(3.4 \%)$ & - \\
\hline Hemoglobin, g/dL & $12.9(11.4-14.3) ; n=279$ & $13.5(12.6-14.8) ; n=107$ & 0.001 \\
\hline Serum creatinine, $\mathrm{mg} / \mathrm{dL}$ & $1.2(0.9-1.5) ; n=329$ & $1.1(0.9-1.3) ; n=130$ & 0.07 \\
\hline
\end{tabular}


Table 2. Cont.

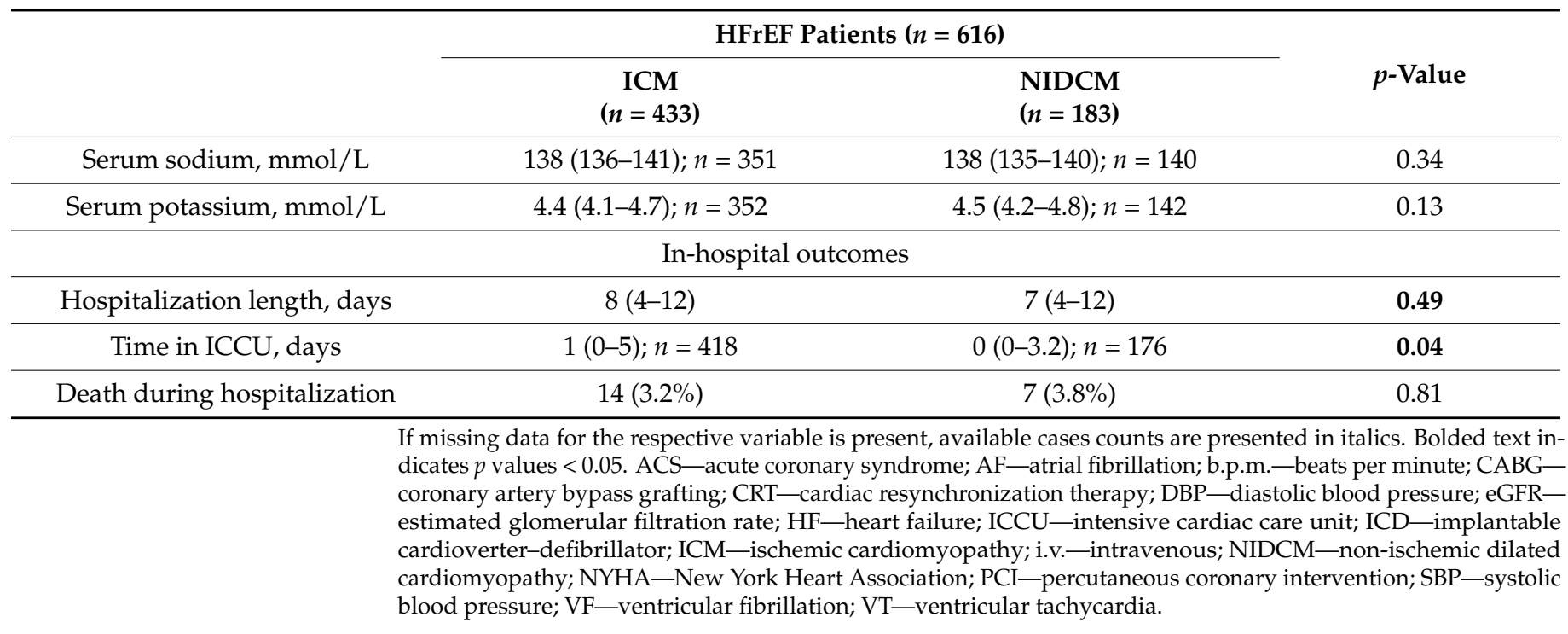

$\mathrm{HF}$ etiology $+\mathrm{NIDCM}+\mathrm{ICM}$

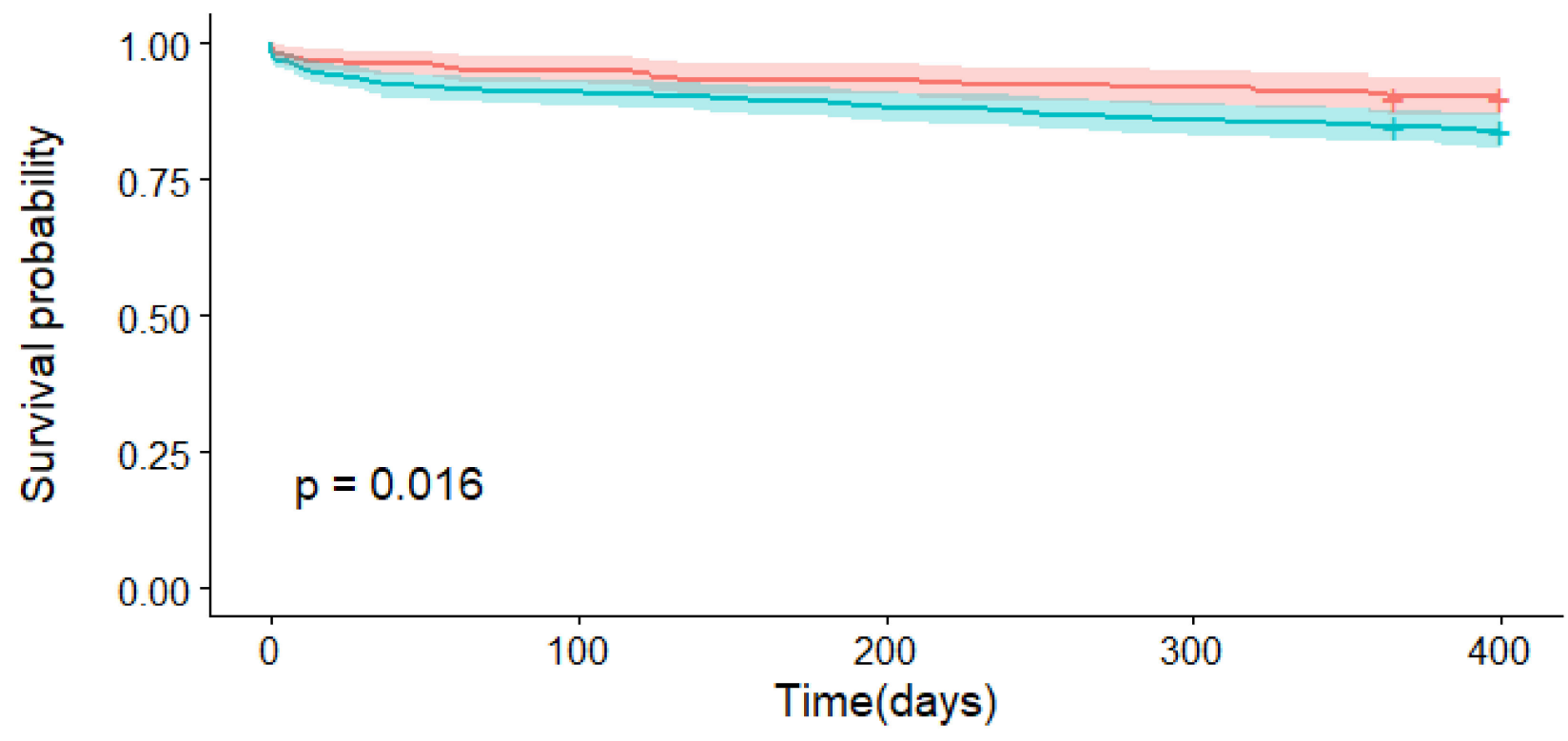

\begin{tabular}{|c|c|c|c|c|c|}
\hline \multicolumn{6}{|c|}{ Number at risk } \\
\hline 음 $\mathrm{NIDCM}$ & \begin{tabular}{|l}
300 \\
555 \\
\end{tabular} & $\begin{array}{l}285 \\
504 \\
\end{array}$ & $\begin{array}{l}280 \\
489 \\
\end{array}$ & $\begin{array}{l}275 \\
476 \\
\end{array}$ & $\begin{array}{l}190 \\
265 \\
\end{array}$ \\
\hline 岌 & 0 & 100 & $\begin{array}{r}200 \\
\text { ne(d }\end{array}$ & 300 & 400 \\
\hline
\end{tabular}

Figure 2. Kaplan-Meier curves for the primary endpoint * of patients with the ICM and NIDCM etiology of HF. ICM—ischemic cardiomyopathy; NIDCM—non-ischemic dilated cardiomyopathy. * Primary endpoint: all-cause death at one year. 


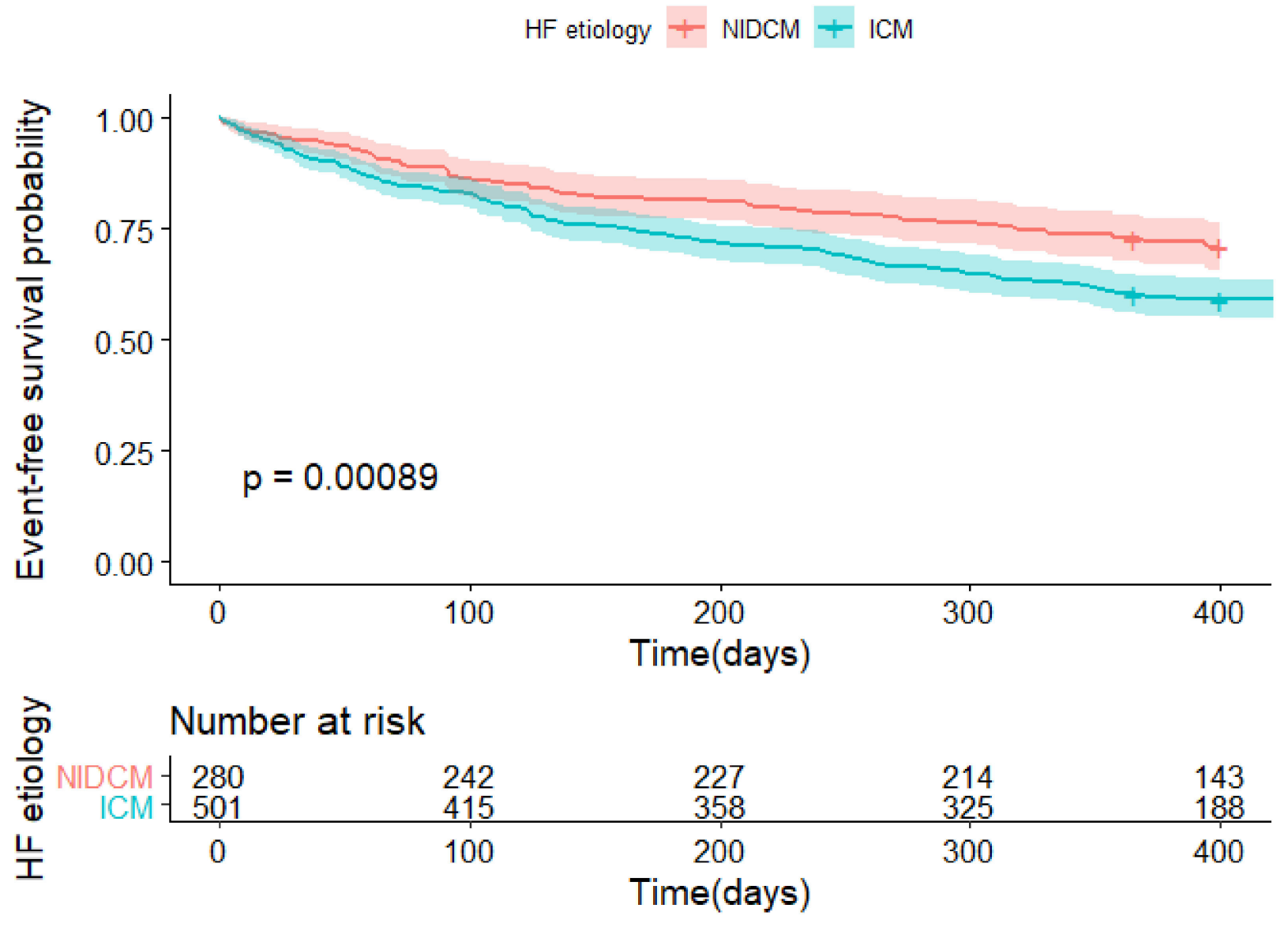

Figure 3. Kaplan-Meier curves for the secondary endpoint* of patients with the ICM and NIDCM etiology of HF. ICM - ischemic cardiomyopathy; NIDCM — non-ischemic dilated cardiomyopathy. * Secondary endpoint: composite of all-cause death and hospitalization for HF worsening at one year).

Table 3. Multivariable analysis of predictors of the primary and secondary endpoints in the total cohort.

\begin{tabular}{ccccccc}
\hline & \multicolumn{3}{c}{ Primary Endpoint } & \multicolumn{3}{c}{ Secondary Endpoint } \\
\hline Variable & HR & CI & $p$-Value & HR & CI & $p$-Value \\
\hline HF etiology as ICM & 1.46 & $0.87-2.47$ & 0.16 & 1.56 & $1.16-2.11$ & $\mathbf{0 . 0 0 3}$ \\
$\begin{array}{c}\text { (NIDCM as reference) } \\
\text { Age, years }\end{array}$ & 1.04 & $1.02-1.06$ & $<\mathbf{0 . 0 0 1}$ & 1.00 & $0.99-1.01$ & 0.72 \\
$\quad$ LVEF, \% & 0.96 & $0.93-0.98$ & $\mathbf{0 . 0 0 3}$ & 0.97 & $0.95-0.99$ & $<\mathbf{0 . 0 0 1}$ \\
$\begin{array}{c}\text { NYHA class, * class IV } \\
\text { or III vs. II or I }\end{array}$ & 1.66 & $1.08-2.54$ & $\mathbf{0 . 0 2}$ & 1.72 & $1.33-2.22$ & $<\mathbf{0 . 0 0 1}$ \\
\hline
\end{tabular}

* At admission to the hospital or first ambulatory visit. Bolded text indicates $p$ values $<0.05$. ICM-ischemic cardiomyopathy; LVEF—left ventricular ejection fraction; NIDCM—non-ischemic dilated cardiomyopathy; NYHANew York Heart Association. 
Table 4. Multivariable analysis of predictors of the primary and secondary endpoints in the ICM and NIDCM groups.

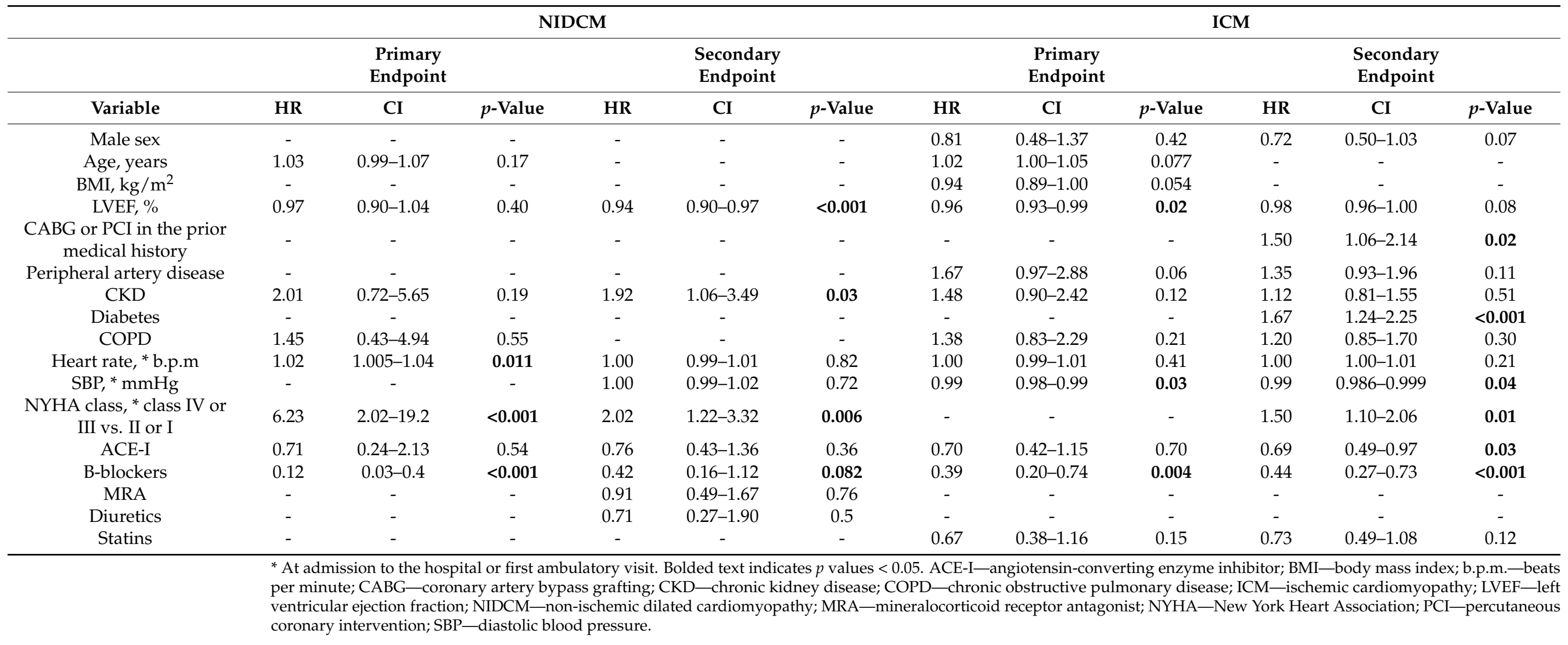




\section{Discussion}

Our study has provided important data on the etiology, associated clinical characteristics and prognoses of real-life patients with HF. The results of this analysis showed that the patients in the ICM group were older and had more comorbidities than the patients in the NIDCM group. The patients with ICM had a higher one-year primary and secondary endpoints occurrence. The ICM etiology was an independent risk factor of the secondary endpoint.

In our study, the ICM etiology was observed in the majority of patients, which is in line with the available data showing ICM as the most common primary etiology of HFrEF, being responsible for $40-70 \%$ of cases [1,6]. In clinical practice, it is important to distinguish between ICM and NIDCM because the diagnosis affects management. The management of ICM patients focuses primarily on the evaluation of the extent of CAD and of the possible indications for revascularization. On the other hand, NIDCM may be caused by multiple factors, e.g., toxic or inflammatory (frequently the exact reason remains undetermined) and may require a multidisciplinary approach [1]. In our study, the patients with the ICM etiology were older and had many more underlying chronic diseases (i.e., chronic kidney disease, diabetes, chronic obstructive pulmonary disease) that are pathophysiologically related to the development and complications of diffused atherosclerosis. Nearly the same patient characteristics of both ICM and NIDCM patients were shown in another real-world patient study [12]. Another finding which should be highlighted is that patients from randomized studies had lower ages and rates of comorbidities [15-18], whereas multimorbidity and older age, being a part of true ICM etiology, significantly influence the clinical presentation and outcomes of patients with HF.

In our study, the ICM patients had significantly worse one-year outcomes than the NIDCM patients. The rates of both all-cause death and all-cause death or HF hospitalization were higher in the ICM than in the NIDCM group, in-line with other real-world data [12], and were higher than in randomized trials [15-18]. What is more, we observed that the ICM etiology was an independent predictor of the secondary endpoint (all-cause death or HF hospitalization). Other predictors of one-year outcomes in ICM patients were similar to those from the previous analyses. Having examined a group of over 3000 patients with LVEF $\leq 40 \%$, Bart et al. observed that the ischemic etiology of cardiomyopathy is an independent predictor of patient death. The authors also noted that the severity of CAD in coronary angiography had a stronger prognostic value than the etiology of cardiomyopathy itself [19]. Similarly, Stevenson et al. identified CAD itself as an independent predictor of mortality and suggested that patients with ischemic HF should be prioritized for heart transplantation [20]. However, these studies were undertaken over twenty years ago, before the era of life-prolonging treatment (i.e., beta-blockers and renin-angiotensin aldosterone system (RAAS) inhibitors, statins, advanced antiplatelet therapy). The ischemic etiology of $\mathrm{HF}$ was also taken into account in the Seattle Heart Failure Model, among various clinical factors used to predict prognosis, mainly in patients with systolic dysfunction [21]. A recently published study showed that ICM etiology adjusted for common risk factors was associated with a higher risk of one- and five-year mortality, as well as HF readmission compared with NIDCM etiology after ICD implantation for primary SCD prevention [12]. It could also be postulated that the extent and severity of CAD offers more prognostic information than the clinical diagnosis of ICM itself. In our previous publication from the ESC-HF Pilot registry on Polish hospitalized patients, we demonstrated that patients with a previous percutaneous coronary intervention (PCI) or coronary artery bypass grafting (CABG) were at a higher risk for death or HF hospitalization than the non-PCI/CABG patients, which could reflect severe CAD and the burden of numerous comorbidities [22].

The NIDCM etiology and its pathophysiology are much more varied and complex than in the case of ICM; therefore, a standard HF pharmacotherapy may not be equally effective (i.e., as in inflammatory cardiomyopathy) [23,24]. However, although the primary mechanism of myocardial injury may be different in patients with ICM and NIDCM, there is an increasing consensus that the progression of $\mathrm{HF}$ is directly related to the activation 
of various neurohormonal systems, particularly the RAAS and the sympathetic nervous systems. It has been demonstrated that proper adherence to guidelines is associated with better survival in patients with HFrEF [25]. Importantly, our patients were treated with a high proportion of life-prolonging HF therapies: $93 \%$ vs. $94 \%, 87 \%$ vs. $93 \%$ and $74 \%$ vs. $82 \%$ of patients received beta-blockers, ACE-I/ARB and aldosterone antagonists in ICM and NIDCM, respectively. The patients were treated similarly or even better than in other studies, including randomized trials $[18,26,27]$. The availability of effective drugs and invasive treatments for ICM should translate into a better prognosis for patients who develop HF due to CAD. Targeted strategies should improve patient care, mainly in ambulatory care, to reduce the rate of readmissions in HF patients.

Electrotherapy with use of ICD and CRT devices is a vital element of HFrEF therapy. ICD reduces the risk of SCD in symptomatic patients with $\mathrm{LVEF} \leq 35 \%$, while CRT reduces morbidity and mortality and improves cardiac function and quality of life [1]. However, there are conflicting results on ICD effectiveness in NIDCM patients. Both ICM and NIDCM patients benefit from ICD implantation for primary prevention of SCD due to the reduction of the death rate as compared to conventional drug therapy. Nevertheless, on average, patients with ICM are at a greater risk of SCD than patients with NIDCM $[1,28]$. Therefore, although the relative benefits are similar, the absolute benefit is greater in patients with ICM $[1,11]$.

Conversely, it is also postulated that the ischemic etiology of HF is less likely to benefit from CRT due to scar tissue [29,30]. However, there is still little evidence that HF etiology affects the effectiveness of CRT and therefore patient outcomes. Current HF ESC guidelines do not account for HF etiology in considerations for CRT implantation [1]. In our study, approximately $30 \%$ and $11 \%$ of patients in both groups had ICD and CRT, respectively, and the rates were similar or higher than in other observational studies [26,27].

Our study shows that even after considering older age, LVEF and NYHA class on admission, the ICM etiology still carried a worse one-year risk of all-cause death and hospitalization for HF than the NIDCM etiology. Further research is needed to refine the predictors of HF treatment response in the ICM and NIDCM patients so that physicians can more accurately personalize patient management to improve outcomes.

\section{Limitations}

The inclusion of real-life patients followed up by cardiologists is an important advantage of the ESC-HF Pilot and ESC-HF-LT registries. The drawbacks include the partial incompleteness of the data and their observational character. What is more, types of etiology were predefined in the case report form and no specific instructions were provided as to how to identify etiology; therefore, the exact cause of NIDCM or the extent of CAD were unknown. It was also not possible to obtain information on the cause of death (dysrhythmia versus pump failure), which also might have been of interest.

\section{Conclusions}

Results from a real-world HF database of patients followed up by cardiologists showed that ICM was present in the majority of HFrEF patients. The patients with ICM were older and had more comorbidities when compared with the patients with NIDCM. The ICM patients had worse one-year outcomes than the NIDCM patients. The ICM etiology was independently associated with a higher risk of the occurrence of all-cause death or HF hospitalization. The identification of the underlying etiology for HFrEF has significant prognostic and therapeutic ramifications.

Supplementary Materials: The following supporting information can be downloaded at: https: / / www.mdpi.com/article/10.3390/biology11020341/s1, Figure S1: Kaplan-Meier curves for the primary endpoint* of patients with the ICM and NIDCM etiology of HF after censoring for in-hospital events; Figure S2. Kaplan-Meier curves for the secondary endpoint* of patients with the ICM and NIDCM etiology of HF after censoring for in-hospital events. 
Author Contributions: A.T., K.O. and A.K.-C. conceived the concept of the study; P.B., M.M., J.D., G.O., M.G., M.G.C.-L., A.P.M. contributed to research design; A.T., K.O., A.W. and E.B. were involved in data collection; A.T., K.O., C.M. analyzed the data. All authors have read and agreed to the published version of the manuscript.

Funding: This research received no external funding.

Institutional Review Board Statement: This is a registry-based study. The study was conducted according to the guidelines of the Declaration of Helsinki. The study protocol was approved by the local ethics committees.

Informed Consent Statement: Informed consent was obtained from all subjects involved in the study.

Data Availability Statement: The data presented in this study are available for three years following the publication on request from the corresponding author.

Conflicts of Interest: The authors declare no conflict of interest.

\section{References}

1. McDonagh, T.A.; Metra, M.; Adamo, M.; Gardner, R.S.; Baumbach, A.; Böhm, M.; Burri, H.; Butler, J.; Čelutkienè, J.; Chioncel, O.; et al. 2021 ESC Guidelines for the diagnosis and treatment of acute and chronic heart failure: Developed by the Task Force for the diagnosis and treatment of acute and chronic heart failure of the European Society of Cardiology (ESC) With the special contribution of the Heart Failure Association (HFA) of the ESC. Eur. Heart J. 2021, 42, 3599-3726. [CrossRef]

2. Parén, P.; Schaufelberger, M.; Björck, L.; Lappas, G.; Fu, M.; Rosengren, A. Trends in prevalence from 1990 to 2007 of patients hospitalized with heart failure in Sweden. Eur. J. Heart Fail. 2014, 16, 737-742. [CrossRef] [PubMed]

3. Gerber, Y.; Weston, S.A.; Redfield, M.M.; Chamberlain, A.M.; Manemann, S.M.; Jiang, R.; Killian, J.M.; Roger, V.L. A Contemporary Appraisal of the Heart Failure Epidemic in Olmsted County, Minnesota, 2000-2010. JAMA Intern. Med. 2015, 175, 996-1004. [CrossRef] [PubMed]

4. Tymińska, A.; Kapłon-Cieślicka, A.; Ozierański, K.; Peller, M.; Balsam, P.; Marchel, M.; Crespo-Leiro, M.G.; Maggioni, A.P.; Jankowska, E.A.; Drożdż, J.; et al. Anemia at Hospital Admission and Its Relation to Outcomes in Patients With Heart Failure (from the Polish Cohort of 2 European Society of Cardiology Heart Failure Registries). Am. J. Cardiol. 2017, 119, $2021-2029$. [CrossRef] [PubMed]

5. Wideqvist, M.; Cui, X.; Magnusson, C.; Schaufelberger, M.; Fu, M. Hospital readmissions of patients with heart failure from real world: Timing and associated risk factors. ESC Heart Fail. 2021, 8, 1388-1397. [CrossRef]

6. Pecini, R.; Møller, D.V.; Torp-Pedersen, C.; Hassager, C.; Køber, L. Heart failure etiology impacts survival of patients with heart failure. Int. J. Cardiol. 2011, 149, 211-215. [CrossRef]

7. Haugaa, K.H.; Tilz, R.; Boveda, S.; Dobreanu, D.; Sciaraffia, E.; Mansourati, J.; Papiashvili, G.; Dagres, N. Implantable cardioverter defibrillator use for primary prevention in ischaemic and non-ischaemic heart disease-indications in the post-DANISH trial era: Results of the European Heart Rhythm Association survey. EP Eur. 2017, 19, 660-664. [CrossRef]

8. Lourenço, C.; Saraiva, F.; Martins, H.; Baptista, R.; Costa, S.; Coelho, L.; Vieira, H.; Monteiro, P.; Franco, F.; Gonçalves, L.; et al. Ischemic versus non-ischemic cardiomyopathy-are there differences in prognosis? Experience of an advanced heart failure center. Rev. Port. Cardiol. 2011, 30, 181-197.

9. Martínez-Sellés, M.; Doughty, R.N.; Poppe, K.; Whalley, G.A.; Earle, N.; Tribouilloy, C.; McMurray, J.J.V.; Swedberg, K.; Køber, L.; Berry, C.; et al. Gender and survival in patients with heart failure: Interactions with diabetes and aetiology. Results from the MAGGIC individual patient meta-analysis. Eur. J. Heart Fail. 2012, 14, 473-479. [CrossRef]

10. Frazier, C.G.; Alexander, K.P.; Newby, L.K.; Anderson, S.; Iverson, E.; Packer, M.; Cohn, J.; Goldstein, S.; Douglas, P.S. Associations of gender and etiology with outcomes in heart failure with systolic dysfunction: A pooled analysis of 5 randomized control trials. J. Am. Coll. Cardiol. 2007, 49, 1450-1458. [CrossRef]

11. Beiert, T.; Straesser, S.; Malotki, R.; Stöckigt, F.; Schrickel, J.W.; Andrié, R.P. Increased mortality and ICD therapies in ischemic versus non-ischemic dilated cardiomyopathy patients with cardiac resynchronization having survived until first device replacement. Arch. Med. Sci. 2019, 15, 845-856. [CrossRef] [PubMed]

12. Higgins, A.Y.; Bjerre, J.; Parzynski, C.S.; Minges, K.E.; Ahmad, T.; Desai, N.R.; Enriquez, A.; Spatz, E.S.; Friedman, D.J.; Curtis, J.P.; et al. Comparison of Mortality and Readmission in Non-Ischemic Versus Ischemic Cardiomyopathy After Implantable Cardioverter-Defibrillator Implantation. Am. J. Cardiol. 2020, 133, 116-125. [CrossRef] [PubMed]

13. Maggioni, A.P.; Dahlström, U.; Filippatos, G.; Chioncel, O.; Leiro, M.C.; Drozdz, J.; Fruhwald, F.; Gullestad, L.; Logeart, D.; Metra, M.; et al. EURObservational Research Programme: The Heart Failure Pilot Survey (ESC-HF Pilot). Eur. J. Heart Fail. 2010, 12, 1076-1084. [CrossRef]

14. Crespo-Leiro, M.G.; Anker, S.D.; Maggioni, A.P.; Coats, A.J.; Filippatos, G.; Ruschitzka, F.; Ferrari, R.; Piepoli, M.F.; Delgado Jimenez, J.F.; Metra, M.; et al. European Society of Cardiology Heart Failure Long-Term Registry (ESC-HF-LT): 1-year follow-up outcomes and differences across regions. Eur. J. Heart Fail. 2016, 18, 613-625. [CrossRef] 
15. Kadish, A.; Dyer, A.; Daubert, J.P.; Quigg, R.; Estes, N.A.M.; Anderson, K.P.; Calkins, H.; Hoch, D.; Goldberger, J.; Shalaby, A.; et al. Prophylactic defibrillator implantation in patients with nonischemic dilated cardiomyopathy. N. Engl. J. Med. 2004, 350, $2151-2158$. [CrossRef] [PubMed]

16. Strickberger, S.A.; Hummel, J.D.; Bartlett, T.G.; Frumin, H.I.; Schuger, C.D.; Beau, S.L.; Bitar, C.; Morady, F. Amiodarone versus implantable cardioverter-defibrillator:randomized trial in patients with nonischemicdilated cardiomyopathy and asymptomaticnonsustained ventricular tachycardia-AMIOVIRT. J. Am. Coll. Cardiol. 2003, 41, 1707-1712. [CrossRef]

17. Bänsch, D.; Antz, M.; Boczor, S.; Volkmer, M.; Tebbenjohanns, J.; Seidl, K.; Block, M.; Gietzen, F.; Berger, J.; Kuck, K.H. Primary Prevention of Sudden Cardiac Death in Idiopathic Dilated Cardiomyopathy. Circulation 2002, 105, 1453-1458. [CrossRef]

18. Køber, L.; Thune, J.J.; Nielsen, J.C.; Haarbo, J.; Videbæk, L.; Korup, E.; Jensen, G.; Hildebrandt, P.; Steffensen, F.H.; Bruun, N.E.; et al. Defibrillator Implantation in Patients with Nonischemic Systolic Heart Failure. N. Engl. J. Med. 2016, 375, 1221-1230. [CrossRef]

19. Bart, B.A.; Shaw, L.K.; McCants, C.B.; Fortin, D.F.; Lee, K.L.; Califf, R.M.; O'Connor, C.M. Clinical Determinants of Mortality in Patients with Angiographically Diagnosed Ischemic or Nonischemic Cardiomyopathy. J. Am. Coll. Cardiol. 1997, 30, 1002-1008. [CrossRef]

20. Stevenson, L.W.; Tillisch, J.H.; Hamilton, M.; Luu, M.; Chelimsky-Fallick, C.; Moriguchi, J.; Kobashigawa, J.; Walden, J. Importance of hemodynamic response to therapy in predicting survival with ejection fraction $\leq 20 \%$ secondary to ischemic or nonischemic dilated cardiomyopathy. Am. J. Cardiol. 1990, 66, 1348-1354. [CrossRef]

21. Levy, W.C.; Mozaffarian, D.; Linker, D.T.; Sutradhar, S.C.; Anker, S.D.; Cropp, A.B.; Anand, I.; Maggioni, A.; Burton, P.; Sullivan, M.D.; et al. The Seattle Heart Failure Model. Circulation 2006, 113, 1424-1433. [CrossRef] [PubMed]

22. Tymińska, A.; Balsam, P.; Ozierański, K.; Peller, M.; Kapłon-Cieślicka, A.; Wancerz, A.; Galas, M.; Marchel, M.; Crespo-Leiro, M.G.; Maggioni, A.P.; et al. Heart failure patients with a previous coronary revascularization: Results from the ESC-HF Registry. Kardiol. Pol. 2018, 76, 144-152. [CrossRef] [PubMed]

23. Tymińska, A.; Ozierański, K.; Caforio, A.L.P.; Marcolongo, R.; Marchel, M.; Kapłon-Cieślicka, A.; Baritussio, A.; Filipiak, K.J.; Opolski, G.; Grabowski, M. Myocarditis and inflammatory cardiomyopathy in 2021: An update. Pol. Arch. Intern. Med. 2021, 131, 594-606. [CrossRef]

24. Balmforth, C.; Simpson, J.; Shen, L.; Jhund, P.S.; Lefkowitz, M.; Rizkala, A.R.; Rouleau, J.L.; Shi, V.; Solomon, S.D.; Swedberg, K.; et al. Outcomes and Effect of Treatment According to Etiology in HFrEF: An Analysis of PARADIGM-HF. JACC Heart Fail. 2019, 7, 457-465. [CrossRef]

25. Komajda, M.; Schöpe, J.; Wagenpfeil, S.; Tavazzi, L.; Böhm, M.; Ponikowski, P.; Anker, S.D.; Filippatos, G.S.; Cowie, M.R.; On behalf of the Qualify Investigators. Physicians' guideline adherence is associated with long-term heart failure mortality in outpatients with heart failure with reduced ejection fraction: The QUALIFY international registry. Eur. J. Heart Fail. 2019, 21, 921-929. [CrossRef] [PubMed]

26. Gajanana, D.; Shah, M.; Junpapart, P.; Romero-Corral, A.; Figueredo, V.M.; Bozorgnia, B. Mortality in systolic heart failure revisited: Ischemic versus non-ischemic cardiomyopathy. Int. J. Cardiol. 2016, 224, 15-17. [CrossRef] [PubMed]

27. Opolski, G.; Ozierański, K.; Lelonek, M.; Balsam, P.; Wilkins, A.; Ponikowski, P.; On Behalf of the Polish Qualify Investigators Adherence to the guidelines on the management of systolic heart failure in ambulatory care in Poland. Data from the international QUALIFY survey. Pol. Arch. Intern. Med. 2017, 127, 657-665. [CrossRef] [PubMed]

28. Kołodziejczak, M.; Andreotti, F.; Kowalewski, M.; Buffon, A.; Ciccone, M.M.; Parati, G.; Scicchitano, P.; Umińska, J.M.; De Servi, S.; Bliden, K.P.; et al. Implantable Cardioverter-Defibrillators for Primary Prevention in Patients With Ischemic or Nonischemic Cardiomyopathy. Ann. Intern. Med. 2017, 167, 103-111. [CrossRef]

29. Cleland, J.G.F.; Mareev, Y.; Linde, C. Reflections on EchoCRT: Sound guidance on QRS duration and morphology for CRT? Eur. Heart J. 2015, 36, 1948-1951. [CrossRef]

30. Linde, C.; Abraham, W.T.; Gold, M.R.; St John Sutton, M.; Ghio, S.; Daubert, C. Randomized trial of cardiac resynchronization in mildly symptomatic heart failure patients and in asymptomatic patients with left ventricular dysfunction and previous heart failure symptoms. J. Am. Coll. Cardiol. 2008, 52, 1834-1843. [CrossRef] 